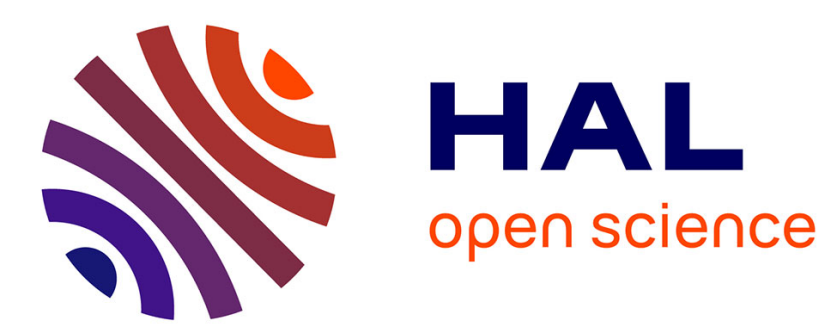

\title{
Lower bounds on joint modulation-estimation performance for the Gaussian MAC
}

Ayşe Ünsal, Raymond Knopp, Neri Merhav

\section{To cite this version:}

Ayşe Ünsal, Raymond Knopp, Neri Merhav. Lower bounds on joint modulation-estimation performance for the Gaussian MAC. IEEE International Symposium on Information Theory (ISIT) 2016, Jul 2016, Barcelona, Spain. 10.1109/ISIT.2016.7541367 . hal-01390980

\section{HAL Id: hal-01390980 \\ https://hal.science/hal-01390980}

Submitted on 16 Nov 2016

HAL is a multi-disciplinary open access archive for the deposit and dissemination of scientific research documents, whether they are published or not. The documents may come from teaching and research institutions in France or abroad, or from public or private research centers.
L'archive ouverte pluridisciplinaire HAL, est destinée au dépôt et à la diffusion de documents scientifiques de niveau recherche, publiés ou non, émanant des établissements d'enseignement et de recherche français ou étrangers, des laboratoires publics ou privés. 


\section{Lower Bounds on Joint Modulation-Estimation Performance for the Gaussian MAC}

\author{
Ayşe Ünsal \\ Univ Lyon, INSA Lyon, Inria, CITI, France \\ ayse.unsal@insa-lyon.fr
}

\author{
Raymond Knopp \\ Eurecom, France \\ raymond.knopp@eurecom.fr
}

\author{
Neri Merhav \\ Technion, Israel \\ merhav@ee.technion.il
}

\begin{abstract}
This paper considers the problem of jointly estimating two independent continuous-valued parameters sent over a Gaussian multiple-access channel (MAC) subject to the mean square error (MSE) as a fidelity criterion. We generalize the parameter modulation-estimation analysis techniques proposed by Merhav in 2012 to a two-user multiple-access channel model to obtain outer bounds to the achievable region in the plane of the MSE's of the two user parameters, as well as the achievable region of the exponential decay rates of these MSE's in the asymptotic regime of long blocks.
\end{abstract}

\section{INTRODUCTION}

First consider the classical problem of a single continuousvalued random parameter $U$ encoded into an $N$-dimensional power-limited signal $\mathbf{x}(U)$ and transmitted over an additivewhite Gaussian noise (AWGN) channel [1], [2]. The $N$ dimensional channel output vector, $\mathbf{y}=\mathbf{x}(U)+\mathbf{z}$, where $\mathbf{z}$ is a Gaussian noise vector with i.i.d. components, is used by the receiver to estimate $U$ by an estimator $\hat{U}(\mathbf{y})$. The goal is to derive a lower bound to the $\operatorname{MSE}, \mathbf{E}(U-\hat{U}(\mathbf{y}))^{2}$, that applies to every modulator $\mathbf{x}(\cdot)$ subject to a given power constraint and to every estimator $\hat{U}(\mathbf{y})$ [3, Chapter 8]. More recently in [4], this class of transmission problems was given the name parameter modulation-estimation and will likely become an important mathematical framework to analyze various remote sensing problems that may arise in fifth generation wireless networks. The purpose of this work is to extend the described problem to the model of the Gaussian multiple-access channel, where two independent parameters, denoted by $U_{1}$ and $U_{2}$, are conveyed from two separate encoders and jointly estimated at the receiver. The aim is to derive outer bounds on the best achievable region for the two MSE's associated with any modulators and estimators of these parameters.

The majority of work dealing with this class of problems considers transmission on a continuous-time channel using finite-energy waveforms without any bandwidth constraint. Goblick [1] provided a lower bound which guarantees an MSE of at least $O\left(\exp \left(-2 \mathcal{E} / N_{0}\right)\right)$, where $\mathcal{E}$ is the energy used to convey $U$ and $N_{0} / 2$ is the two-sided power spectral-density of the channel noise process. Goblick provided several examples of parameter-modulation-estimation schemes, although only one achieves what was later known to achieve the best asymptotic performance, namely $\mathbf{E}[\hat{U}-U]^{2} \leq O\left(\exp \left(-\mathcal{E} / 3 N_{0}\right)\right)$. This simple digital scheme is based on first partitioning the parameter space into $M$ equally-spaced bins and transmitting the index of the bin in which the parameter lies to the receiver, using $M$-ary orthogonal modulation. Another parameter-modulation strategy in continuous-time is given in [3, pp. 623] where the parameter is reflected in the delay of a purely analog signaling pulse sent across the channel. When the bandwidth of the pulse is allowed to grow without bound, this scheme achieves the same exponential behaviour as Goblick's scheme and also provides a link with the classical ranging problem where the objective is to estimate the random delay of an incoming waveform perturbed by Gaussian noise [5]. In [2], Wyner and Ziv show that Goblick's lower bound can be improved to $O\left(\exp \left(-\mathcal{E} / 2 N_{0}\right)\right)$. The works in [6]-[8] further improve the factor in the denominator of the exponential function's argument progressively from 2.889 to 2.896 and finally to 2.970 , virtually closing the gap to Goblick's practical scheme. The main contribution of [4] is the characterization of the parameter modulation-estimation problem for finitedimensional transmission over the continuous-time AWGN channel. In this paper we consider a discrete-time version of the problem in [4] for a two-user discrete-time AWGN MAC with independent parameters. The MAC model is important for the study of the physical layer of the so-called Internet of Things (IoT). The IoT will include energy-constrained objects that provide low-periodicity sensory information to data centers via the uplink channel of fifth generation cellular networks. A recent example of a similar scenario can be found in [9], [10], where using an information-theoretic approach lower bounds on the MSE region are provided for the transmission of two correlated analog source samples with and without causal feedback on a discrete-time AWGN MAC without a constraint on the number of signaling dimensions.

The next section describes the system model and problem formulation. In Section III, we first study the $\left(\mathrm{MSE}_{1}, \mathrm{MSE}_{2}\right)$ region, where $\mathrm{MSE}_{1}$ and $\mathrm{MSE}_{2}$ are associated to the parameter estimates, using a generalization of Shannon's zerorate lower bound [11] for the two-user discrete-time MAC which allows us to characterize the MSE region in terms of the signal energies without any limitation on the signal dimensions. We note that we have currently opted for a simpler bounding technique for the MAC than those considered in [6][8] since they rely on the continuous-time infinite-bandwidth assumption and are not directly applicable in discrete-time. We also consider the exponential behaviour of $\left(\mathrm{MSE}_{1}, \mathrm{MSE}_{2}\right)$ by characterizing an inner-bound to the region of achievable MSE exponents for any joint parameter-modulation estimation scheme. To this end, we adapt the multiple-access results of [12] to the discrete-time AWGN channel. To find the tightest characterization, we also use the bounds on the Gaussian reliability function proposed in [11] and the improved bounds from [13] coupled with the results from [14] which provides the means to make use of single-user error-exponents for the characterization of multiuser channels. Finally, in Section IV, we draw conclusions from our results.

\section{SySTEM MOdEL}

We consider an arbitrary parameter modulation-estimation scheme for two random variables $U_{1}$ and $U_{2}$ which are independent and uniformly distributed over the interval $[0,1)$. They are conveyed by distinct modulators, $\mathbf{x}_{1}\left(U_{1}\right)$ and $\mathbf{x}_{2}\left(U_{2}\right)$ over an $N$-dimensional real-valued AWGN MAC obeying the 
following signal model

$$
\mathbf{y}=\mathbf{x}_{1}\left(U_{1}\right)+\mathbf{x}_{2}\left(U_{2}\right)+\mathbf{z}
$$

where $\mathbf{z}$ represents the Gaussian channel noise. The modulators are constrained in energy as

$$
\left\|\mathbf{x}_{j}\left(U_{j}\right)\right\|^{2} \leq N \mathcal{S}_{j}=\mathcal{E}_{j}, \forall U_{j}, \text { for } j=1,2
$$

and the covariance matrix of the noise is $\mathbf{E z z}^{t}=\sigma^{2} \mathbf{I}_{N}$. At the receiver we consider estimators $\hat{U}_{j}(\mathbf{y})$ with corresponding $\mathrm{MSE}_{j}=\mathbf{E}\left(U_{j}-\hat{U}_{j}(\mathbf{y})\right)^{2}$. The objective of the next section is to provide different characterizations of the regions which dictate achievable $\left(\mathrm{MSE}_{1}, \mathrm{MSE}_{2}\right)$ pairs for any choice of modulators and estimators of $\left(U_{1}, U_{2}\right)$. The first characterization does not impose any limit on the dimensionality $N$ and provides a direct characterization of $\left(\mathrm{MSE}_{1}, \mathrm{MSE}_{2}\right)$. The second assumes finite $N$ and defines the region in terms of the exponents $\left(\epsilon_{1}, \epsilon_{2}\right)$ where

$$
\epsilon_{j} \triangleq \lim _{N \rightarrow \infty} \frac{1}{N} \log \mathbf{E}\left[\hat{U}_{j}-U_{j}\right]^{2}, \text { for } j=1,2 .
$$

\section{LOWER BOUNDS ON THE MSE}

In this part, we firstly recall the single-user approach from [4] and improve the lower bound on the MSE for any parameter-modulator scheme without any constraint on the number of signalling dimensions $N$, which will subsequently be used in the corresponding bound for two modulators on the MAC.

\section{A. An improved lower bound for the single-user case}

Let $\hat{U}$ denote the estimate of uniformly distributed $U$ over the interval $[0,1)$. It is shown that for the single-user problem described in Section I, the probability of the estimation error $|\hat{U}-U|$ to exceed some function of a positive parameter $\Delta$ is lower bounded as $\operatorname{Pr}\{|\hat{U}-U|>\Delta / 2\} \geq L_{B}(\Delta)$ [4, eq. 21]. Here we have the following generalized hypothesis testing problem with $M$ equiprobable hypothesis,

$$
\mathcal{H}_{i}: \mathbf{y}_{\text {single }}=\mathbf{x}(u+i \Delta)+\mathbf{z}
$$

for $i \in\{1, \cdots, M\}$ where the nuisance parameter $u \in$ $[0,1-(M-1) \Delta) . L_{B}(\Delta)$ is found by combining the ZivZakai approach with any lower bound on the average probability of error for an arbitrary encoding function of a given rate. The probability of error of the optimal (ML) detector in the $M$ ary hypothesis problem, denoted by $P_{e}(u, \Delta)=\operatorname{Pr}(\hat{i} \neq i \mid u)$, is bounded as follows.

$$
\begin{aligned}
& \int_{0}^{1-(M-1) \Delta} d u \cdot P_{e}(u, \Delta) \\
& \leq \frac{1}{M} \sum_{i=0}^{M-1} \int_{0}^{1-(M-1) \Delta} \mathrm{d} u \cdot \operatorname{Pr}\left\{|\hat{U}-U|>\frac{\Delta}{2} \mid U=u+i \Delta\right\} \\
& \stackrel{(a)}{=} \frac{1}{M} \sum_{i=0}^{M-1} \operatorname{Pr}\left\{|\hat{U}-U|>\frac{\Delta}{2}, i \Delta \leq U \leq 1-(M-1) \Delta+i \Delta\right. \\
& =\frac{1}{M} \operatorname{Pr}\{|\hat{U}-U|>\Delta / 2\} .
\end{aligned}
$$

We note that (5) is valid for all $M$ and $\Delta$ such that $(M-1) \Delta<1$. If we add the condition that $M \Delta>1$, which amounts to $1 / \Delta<M<1+(1 / \Delta)$ or equivalently $M=\lceil 1 / \Delta\rceil$, the intervals in step (a) become disjoint and cover the entire range $0 \leq U \leq 1$, i.e. for $i=M-1, U$ cannot exceed 1 and for $i=0, U \geq 0$. This bound is related to the MSE as

$$
\begin{aligned}
& \int_{0}^{1} d \Delta \cdot \Delta \cdot \operatorname{Pr}\{|\hat{U}-U|>\Delta / 2\} \\
& \stackrel{(a)}{\leq} 4 \int_{0}^{1} d \delta \cdot \delta \cdot \operatorname{Pr}\{|\hat{U}-U|>\delta\} \stackrel{(b)}{=} 2 \mathbf{E}[\hat{U}-U]^{2} .
\end{aligned}
$$

In step (a) the integration variable is changed according to $\delta=\Delta / 2$ and the integration interval is stretched to $[0,1)$ whereas in (b) the following identity is used $\mathbf{E}[\hat{U}-U]^{2}=2 \int_{0}^{1} d \Delta \cdot \Delta \cdot \operatorname{Pr}\{|\hat{U}-U|>\Delta\}$. Let us denote $\mathbf{E}[\hat{U}-U]^{2}$ by $\mathrm{MSE}_{\mathrm{s}}$, combining (5) with (6), the improved single-user lower bound is given by

$$
\begin{aligned}
& \mathrm{MSE}_{\mathrm{s}} \geq \frac{1}{2} \int_{0}^{1} d \Delta\lceil 1 / \Delta\rceil \Delta(1+\Delta-\Delta\lceil 1 / \Delta\rceil) P_{\mathrm{ZR}}(\mathcal{E},\lceil 1 / \Delta\rceil) \\
& =1 / 2 \sum_{i=2}^{\infty} \int_{1 / i}^{1 /(i-1)} d \Delta\left(\Delta i+\Delta^{2} i-\Delta^{2} i^{2}\right) P_{\mathrm{ZR}}(\mathcal{E}, i) \\
& =1 / 2 \sum_{i=2}^{\infty} \frac{3 i-2}{6 i^{2}(i-1)^{2}} P_{\mathrm{ZR}}(\mathcal{E}, i)
\end{aligned}
$$

where $P_{\mathrm{ZR}}(\mathcal{E}, i)=\frac{1}{i} \sum_{m=2}^{i} Q\left(\sqrt{\frac{m}{m-1} \frac{\mathcal{E}}{2 \sigma^{2}}}\right)$ [11]. Note that, resulting bound on the error probability (5) is $M$ times larger than the original result given by [4, eq. (21)] which improves (7) in terms of the factor in front of the exponent.

\section{B. Lower bounds on any linear combinations of the MSE's in a two-user MAC}

For the joint estimation of $\left(U_{1}, U_{2}\right)$, we have the following generalized hypothesis testing problem,

$$
\mathcal{H}_{i, i^{\prime}}: \mathbf{y}=\mathbf{x}_{1}\left(u_{1}+i \Delta_{1}\right)+\mathbf{x}_{2}\left(u_{2}+i^{\prime} \Delta_{2}\right)+\mathbf{z},
$$

for $i \in\left\{1, \cdots, M_{1}\right\}$ and $i^{\prime} \in\left\{1, \cdots, M_{2}\right\}$ where $u_{1} \in$ $\left[0,1-\left(M_{1}-1\right) \Delta_{1}\right), u_{2}$ is similarly in a bin of size $\Delta_{2}$, $u_{2} \in\left[0,1-\left(M_{2}-1\right) \Delta_{2}\right)$. Both $u_{1}$ and $u_{2}$ should be seen as nuisance parameters unknown to the receiver. We will consider two types of results using the above problem. The first corresponds to fixed values of $M_{1}$ and $M_{2}$ (and $\Delta_{1}, \Delta_{2}$ ), that will yield non-asymptotic results on the MSE's themselves (for any $N$ ). The other type of results refers to the asymptotic regime of a long block $N$, where $M_{1}$ and $M_{2}$ are allowed to grow exponentially with $N$, at arbitrary rates to be optimized, and our asymptotic results concern the asymptotic exponential rates of the two MSE's. We denote the conditional probability of error as a function of $\left(u_{1}, u_{2}\right)$ by $P_{e}\left(u_{1}, u_{2}, \Delta_{1}, \Delta_{2}\right)=$ $\operatorname{Pr}\left(\left(\hat{i}, \hat{i^{\prime}}\right) \neq\left(i, i^{\prime}\right) \mid u_{1}, u_{2}\right)$ where the overall probability of error is $P_{e}=\int_{u_{1}} d u_{1} p\left(u_{1}\right) \int_{u_{2}} d u_{1} p\left(u_{2}\right) P_{e}\left(u_{1}, u_{2}, \Delta_{1}, \Delta_{2}\right)$ with $p($.$) being the probability density function. A lower bound$ on $P_{e}\left(u_{1}, u_{2}\right)$ can be derived by generalizing Shannon's zerorate lower bound for an AWGN MAC. The overall probability of error for this channel can be decomposed in three terms as

$$
\left\{\begin{array}{l}
P_{e}=\operatorname{Pr}\left(\hat{i}=i, \hat{i^{\prime}} \neq i^{\prime} \mid u_{1}, u_{2}\right)+ \\
\operatorname{Pr}\left(\hat{i} \neq i, \hat{i^{\prime}}=i^{\prime} \mid u_{1}, u_{2}\right)+\operatorname{Pr}\left(\hat{i} \neq i, \hat{i^{\prime}} \neq i^{\prime} \mid u_{1}, u_{2}\right) .
\end{array}\right.
$$

Shannon's bound [11, eq. (80)] is based on first computing the average squared Euclidean distance between pairs of modulated signals which should be done for each of the terms corresponding to the three types of error events in (9). In the first term of (9) there are $M_{2}\left(M_{2}-1\right)$ possible signal pairs and so the average squared Euclidean distance between all such pairs is $D_{2}^{2}\left(u_{1}, u_{2}\right) \leq 2 M_{2} \mathcal{E}_{2} /\left(M_{2}-1\right)$. Similarly, 
the average squared Euclidean distance for the second term of (9) is bounded as $D_{1}^{2}\left(u_{1}, u_{2}\right) \leq 2 M_{1} \mathcal{E}_{1} /\left(M_{1}-1\right)$ with $M_{1}\left(M_{1}-1\right)$ possible signal pairs for this user. For the third term, there are $M_{1} M_{2}\left(M_{1}-1\right)\left(M_{2}-1\right)$ possible pairs that differ in both indices, so that we have

$$
D_{12}^{2}\left(u_{1}, u_{2}\right) \leq 2 M_{1} \mathcal{E}_{1} /\left(M_{1}-1\right)+2 M_{2} \mathcal{E}_{2} /\left(M_{2}-1\right) \text {. }
$$

The proofs of $D_{1}^{2}\left(u_{1}, u_{2}\right), D_{2}^{2}\left(u_{1}, u_{2}\right)$ and $D_{12}^{2}\left(u_{1}, u_{2}\right)$ are omitted here due to space constraints. By progressively removing points at the average distance as in Shannon's original bound [11, eq. 81], we obtain the overall bound on (9) as follows.

$$
\begin{aligned}
& P_{e} \geq P_{Z R}\left(\mathcal{E}_{1}, \mathcal{E}_{2}, M_{1}, M_{2}\right) \\
& =\frac{1}{M_{1}} \sum_{m=2}^{M_{1}} Q\left(\sqrt{\frac{m}{m-1} \frac{\mathcal{E}_{1}}{2 \sigma^{2}}}\right)+\frac{1}{M_{2}} \sum_{m=2}^{M_{2}} Q\left(\sqrt{\frac{m}{m-1} \frac{\mathcal{E}_{2}}{2 \sigma^{2}}}\right) \\
& +\frac{1}{M_{1} M_{2}} \sum_{m_{1}=2}^{M_{1}} \sum_{m_{2}=2}^{M_{2}} Q\left(\sqrt{\frac{m_{1}}{m_{1}-1} \frac{\mathcal{E}_{1}}{2 \sigma^{2}}+\frac{m_{2}}{m_{2}-1} \frac{\mathcal{E}_{2}}{2 \sigma^{2}}}\right) .
\end{aligned}
$$

In a two-user MAC, the error is defined as either one of the sources being in error. In this case, we have an extended version of the lower bound $L_{B}(\Delta)$ using the positive parameters $\Delta_{1}, \Delta_{2}$ for $U_{1}, U_{2}$, respectively as

$\operatorname{Pr}\left\{\left|\hat{U}_{1}-U_{1}\right|>\Delta_{1} / 2\right.$ or $\left.\left|\hat{U}_{2}-U_{2}\right|>\Delta_{2} / 2\right\} \geq L_{B}\left(\Delta_{1}, \Delta_{2}\right)$, where the left-hand side (1.h.s.) is further upper bounded by the union bound to yield

$$
\operatorname{Pr}\left\{\left|\hat{U}_{1}-U_{1}\right|>\Delta_{1} / 2\right\}+\operatorname{Pr}\left\{\left|\hat{U}_{2}-U_{2}\right|>\Delta_{2} / 2\right\} \geq L_{B}\left(\Delta_{1}, \Delta_{2}\right) \text {. }
$$

As for upper bounding the error probability $P_{e}$, we have

$$
\begin{gathered}
\int_{0}^{1-\left(M_{1}-1\right) \Delta_{1}} d u_{1} \cdot p\left(u_{1}\right) \int_{0}^{1-\left(M_{2}-1\right) \Delta_{2}} d u_{2} \cdot p\left(u_{2}\right) \\
\leq \frac{\left(\operatorname{Pr}\left\{\left|\hat{U}_{1}-U_{1}\right|>\Delta_{1} / 2\right\}+\operatorname{Pr}\left\{\left|\hat{U}_{2}-U_{2}, u_{2}\right|>\Delta_{2} / 2\right\}\right)}{\left\lceil 1 / \Delta_{1}\right\rceil\left\lceil 1 / \Delta_{2}\right\rceil}
\end{gathered}
$$

which is simply the generalization of (5) to the MAC problem. Step (a) of the upper bound (13) is obtained by introducing the condition of $M_{j} \Delta_{j}>1$, which is equivalent to $M_{j}=\left\lceil 1 / \Delta_{j}\right\rceil$, for $j=1,2$. We note that (13) is valid for all $M_{j}$ and $\Delta_{j}$ such that $\left(M_{j}-1\right) \Delta_{j}<1$. Combining (12) and (13) with (11), we finally have

$$
\begin{gathered}
L_{B}\left(\Delta_{1}, \Delta_{2}\right) \geq\left(\left\lceil 1 / \Delta_{1}\right\rceil\left\lceil 1 / \Delta_{2}\right\rceil\right)\left(1+\Delta_{1}-\left\lceil 1 / \Delta_{1}\right\rceil \Delta_{1}\right) \\
\left(1+\Delta_{2}-\left\lceil 1 / \Delta_{2}\right\rceil \Delta_{2}\right) P_{Z R}\left(\mathcal{E}_{1}, \mathcal{E}_{2},\left\lceil 1 / \Delta_{1}\right\rceil,\left\lceil 1 / \Delta_{2}\right\rceil\right) .
\end{gathered}
$$

We now state the first main result.

Theorem 1. For arbitrary parameter-modulators $\mathbf{x}_{j}\left(U_{j}\right), \quad j=1,2$ transmitting over a two-user Gaussian $M A C$ defined by (1) and a fixed free parameter $\theta \in[0,1)$, we have that

$$
\mathrm{MSE}_{j} \geq \max \left(\mathrm{MSE}_{\mathrm{s}, j}, \max _{0<\theta \leq 1}\left[C(\theta) / 2-\mathrm{MSE}_{3-j} / \theta^{2}\right]\right),
$$

where $\mathrm{MSE}_{\mathrm{s}, j}$ denotes the lower bound on the MSE in estimating the corresponding source in an AWGN point-to-point channel given by (7), with $C(\theta)=\int_{0}^{1} d \Delta \cdot \Delta \cdot L_{B}(\Delta, \theta \Delta)$ and $L_{B}(.,$.$) is given by (14).$
Proof. Letting $\Delta_{2}=\theta \Delta$ and $\Delta_{1}=\Delta$ and integrating both sides of (12) w.r.t. $\Delta$ yields

$$
\begin{gathered}
\int_{0}^{1} d \Delta \cdot \Delta\left(\operatorname{Pr}\left\{\left|\hat{U}_{1}-U_{1}\right|>\Delta / 2\right\}+\operatorname{Pr}\left\{\left|\hat{U}_{2}-U_{2}\right|>\theta \Delta / 2\right\}\right) \\
\geq C(\theta),
\end{gathered}
$$

which is further bounded as

$$
\mathrm{MSE}_{1}+\frac{\mathrm{MSE}_{2}}{\theta^{2}} \geq \frac{C(\theta)}{2} .
$$

Optimizing over $\theta$ provides the second term of the maximization in (15). $\mathrm{MSE}_{1}$ must also be limited by the single-user bound in (7) since this corresponds to the case where $U_{2}$ is known to the receiver and where $\mathbf{x}_{2}\left(U_{2}\right)$ can be removed from y. By symmetry the same lower bound could be given for $\mathrm{MSE}_{2}$ by reversing the roles of both sources.

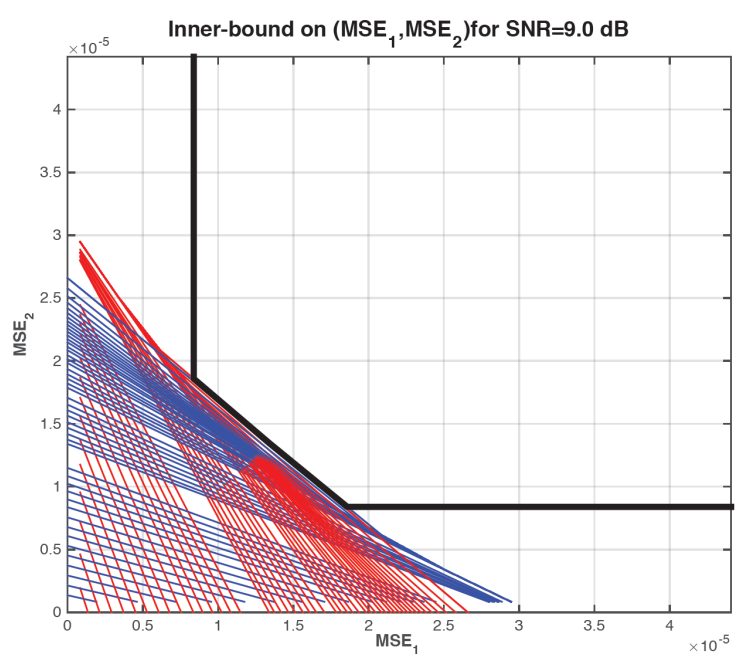

Figure 1. Numerical evaluation of (15) for $\mathrm{SNR}=9 \mathrm{~dB}$ with all possible values of $\theta$.

In Figure 1, we present numerical evaluation of (15) for different values of $\theta$. Note that signal-to-noise ratio (SNR) is defined by $\mathcal{E}_{j} / \sigma^{2}$ for $j=1,2$. The wall and floor, the vertical and horizontal parts of the black curve to the axes, correspond to $\mathrm{MSE}_{\mathrm{s}, j}$. The red and blue curves represent all possible bounds for $\theta \in[0,1)$. The convex hull is depicted in solid black curve.

\section{Lower Bounds on the MSE exponents}

This subsection deals with modifying the form of the lower bound presented in Theorem 1 in order to derive an outer bound of the achievable region of the MSE exponents defined by (3) in Section II.

Theorem 2. For arbitrary $N$-dimensional parametermodulators $\mathbf{x}_{j}\left(U_{j}\right), j=1,2$ transmitting over a two-user Gaussian MAC defined by (1) and for $R \geq 0$ and $\alpha \geq 0$, the MSE exponent $\epsilon_{j}$ for $j=1,2$ is bounded below as

$$
\epsilon_{j} \geq-\inf _{\alpha: F(\alpha)+2 \alpha \leq-\epsilon_{3-j}} F(\alpha)
$$

where $\left.F(\alpha) \triangleq \min _{R}\left[E_{u}(R, R+\alpha)+2 R\right]\right\}$ and $E_{u}\left(R_{1}, R_{2}\right)$ is any upper bound on the error exponent of the two-user Gaussian MAC with codebooks of rate $R_{1}$ and $R_{2}$. 
Proof. Substituting $\Delta=e^{-R N}$ and $\theta=e^{-\alpha N}$ into (17) and changing the integration variable on the r.h.s. of (17) to $R$, we obtain

$\mathrm{MSE}_{1}+e^{2 \alpha N} \mathrm{MSE}_{2} \geq \frac{N}{2} \int_{0}^{\infty} d R \cdot e^{-2 R N} \cdot L_{B}\left(e^{-R N}, e^{-(R+\alpha) N}\right)$.

From the Laplace approximation the right-hand side (r.h.s.) is on the order of $\exp \left\{-N \min _{R}\left[E_{u}(R, R+\alpha)+2 R\right]\right\}=$ $\exp \{-N F(\alpha)\}$. Similarly, the 1.h.s. is on the order of $\exp \left\{\max \left\{\epsilon_{1}, \epsilon_{2}+2 \alpha\right\}\right\}$. Thus, we obtain

$$
\max \left\{\epsilon_{1}, \epsilon_{2}+2 \alpha\right\} \geq-F(\alpha) \quad \forall \alpha \geq 0 .
$$

In other words, for every $\alpha \geq 0$, there exists $\lambda \in[0,1]$ such that $\lambda \epsilon_{1}+(1-\lambda)\left(\epsilon_{2}+2 \alpha\right) \geq-F(\alpha)$ or equivalently:

$$
\begin{aligned}
& \epsilon_{1} \geq-\inf _{\alpha \geq 0} \sup _{0 \leq \lambda \leq 1} \frac{F(\alpha)+(1-\lambda)\left(\epsilon_{2}+2 \alpha\right)}{\lambda} \\
&=-\inf _{\alpha: F(\alpha)+2 \alpha \leq-\epsilon_{2}} F(\alpha) .
\end{aligned}
$$

For $j=2$, the roles of $\epsilon_{1}$ and $\epsilon_{2}$ are switched and $F(\alpha)$ is redefined as $\min _{R}\left[E_{u}(R+\alpha, R)+2 R\right]$.

For the purpose of numerical evaluation, we will study three different bounds on the error exponent in (18) to be used for $E_{u}\left(R_{1}, R_{2}\right)$.

1) Divergence bound: $E_{u}\left(R_{1}, R_{2}\right)$ is chosen as the spherepacking bound of [12], taking the auxiliary channel $W$ to be a Gaussian MAC with noise variance $\sigma_{w}^{2}$. For inputs of powers as defined by (2), the rate region of the auxiliary Gaussian MAC $W$ is given by

$$
\begin{aligned}
R_{j} & \leq \frac{1}{2} \log \left(1+\frac{\mathcal{S}_{j}}{\sigma_{w}^{2}}\right) \quad j=1,2 \\
R_{1}+R_{2} & \leq \frac{1}{2} \log \left(1+\frac{\mathcal{S}_{1}+\mathcal{S}_{2}}{\sigma_{w}^{2}}\right)
\end{aligned}
$$

which implies that for $W$ to exclude $\left(R_{1}, R_{2}\right)$ from the achievable region,

$$
\begin{aligned}
\sigma_{w}^{2} & \geq \min \left\{\frac{\mathcal{S}_{1}}{e^{2 R_{1}}-1}, \frac{\mathcal{S}_{2}}{e^{2 R_{2}}-1}, \frac{\mathcal{S}_{1}+\mathcal{S}_{2}}{e^{2\left(R_{1}+R_{2}\right)}-1}\right\} \\
& \triangleq \sigma_{0}^{2}\left(R_{1}, R_{2}\right)
\end{aligned}
$$

and it is assumed that $\sigma_{0}^{2}\left(R_{1}, R_{2}\right)>\sigma^{2}$. Thus,

$$
\begin{aligned}
& E_{s p}\left(R_{1}, R_{2}\right)=\frac{1}{2}\left[\frac{\sigma_{0}^{2}\left(R_{1}, R_{2}\right)}{\sigma^{2}}-\ln \left(\frac{\sigma_{0}^{2}\left(R_{1}, R_{2}\right)}{\sigma^{2}}\right)-1\right] \\
& =\min \left\{D\left(R_{1}, \mathcal{S}_{1}\right), D\left(R_{2}, \mathcal{S}_{2}\right), D\left(R_{1}+R_{2}, \mathcal{S}_{1}+\mathcal{S}_{2}\right)\right\}
\end{aligned}
$$

where the divergence function is defined using [12, eq. (5.27)] as

$$
D(R, \mathcal{S}) \triangleq \frac{1}{2}\left[\frac{\mathcal{S}}{\sigma^{2}\left(e^{2 R}-1\right)}-\ln \left(\frac{\mathcal{S}}{\sigma^{2}\left(e^{2 R}-1\right)}\right)-1\right] .
$$

We first need to calculate

$$
\begin{aligned}
& F(\alpha)=\inf _{R>0}\left\{2 R+E_{s p}(R, R+\alpha)\right\} \\
& =\inf _{R>0}\left\{2 R+\frac{1}{2}\left[\frac{\sigma_{0}^{2}(R, R+\alpha)}{\sigma^{2}}-\ln \left(\frac{\sigma_{0}^{2}(R, R+\alpha)}{\sigma^{2}}\right)-1\right]\right\} \\
& =\min \left\{F_{1}, F_{2}(\alpha), F_{12}(\alpha)\right\}
\end{aligned}
$$

with

$$
\begin{aligned}
& F_{1}=\inf _{R \geq 0}\left[2 R+D\left(R, \mathcal{S}_{1}\right)\right] \\
& F_{2}(\alpha)=\inf _{R \geq 0}\left[2 R+D\left(R+\alpha, \mathcal{S}_{2}\right)\right] \\
& F_{12}(\alpha)=\inf _{R \geq 0}\left[2 R+D\left(2 R+\alpha, \mathcal{S}_{1}+\mathcal{S}_{2}\right)\right] .
\end{aligned}
$$

The channel rates that minimize the three exponents $F_{1}, F_{2}(\alpha)$ and $F_{12}(\alpha)$ given by (28)-(30) are denoted respectively by $R_{1}^{*}$, $R_{2}^{*}$ and $R_{12}^{*}$. Using these rate functions we can reformulate the minimum functions $F_{1}^{*}, F_{2}^{*}(\alpha)$ and $F_{12}^{*}(\alpha)$ as functions of $R_{1}^{*}$, $R_{2}^{*}$ and $R_{12}^{*}$, respectively. Considering the constraint in (18), we choose the largest $\alpha$ satisfying

$$
-\epsilon_{2} \geq \min \left\{F_{1}^{*}, F_{2}^{*}(\alpha), F_{12}^{*}(\alpha)\right\}+2 \alpha .
$$

The constraint $-\epsilon_{2} \geq F_{1}^{*}+2 \alpha$ yields

$$
\alpha \leq-\frac{\epsilon_{2}+F_{1}^{*}}{2} \triangleq \alpha_{1}\left(\epsilon_{2}\right) \text {. }
$$

The constraint $-\epsilon_{2} \geq F_{2}^{*}(\alpha)+2 \alpha$ gives no requirement concerning $\alpha$, it is simply the single-user bound for the second user. For the two-user component $-\epsilon_{2} \geq F_{12}^{*}(\alpha)+2 \alpha$ we have

$$
\alpha \leq-\frac{1}{2}\left(F_{12}^{*}(\alpha)-\epsilon_{2}\right) \triangleq \alpha_{2}\left(\epsilon_{2}\right) .
$$

Thus, the constraint becomes

$$
\alpha \leq \alpha^{*}\left(\epsilon_{2}\right) \triangleq \max \left\{\alpha_{1}\left(\epsilon_{2}\right), \alpha_{2}\left(\epsilon_{2}\right)\right\},
$$

resulting in the overall lower bound

$$
\epsilon_{1} \geq-F\left[\alpha^{*}\left(\epsilon_{2}\right)\right]=-\min \left\{F_{1}, F_{2}\left[\alpha^{*}\left(\epsilon_{2}\right)\right], F_{12}\left[\alpha^{*}\left(\epsilon_{2}\right)\right]\right\} .
$$

The roles of the users should be interchanged to obtain the outer bound for $\epsilon_{2}$ as a function of $\epsilon_{1}$. The overall outer bound on the achievable region of the MSE exponents is the intersection of the two.

2) Shannon's sphere-packing bound: For rates confined to $[0, \mathcal{C})$ where $\mathcal{C}$ is the channel capacity defined by $\mathcal{C}=$ $(1 / 2) \log (1+A)$ and $A$ represents the SNR defined by $\mathcal{S} / \sigma^{2}$. Shannon's sphere-packing bound in a point-to-point channel $E_{s p}(\psi(R), A)$ is an upper bound on the Gaussian reliability function $E(R, A)$ [11]. The sphere-packing bound is given by

$$
E_{s p}(\psi, A)=\frac{A}{2}-\frac{\sqrt{A} g(\psi, A) \cos \psi}{2}-\log (g(\psi, A) \sin \psi)
$$

where $g(\psi, A)=1 / 2\left(\sqrt{A} \cos \psi+\sqrt{A \cos ^{2} \psi+4}\right)$ and $\psi(R)=\arcsin e^{-R}$. The only positive and real root that satisfies $\min _{R}\left(E_{s p}(\psi(R), A)+2 R\right)$ is

$$
R_{\text {min }}=\log \left\{\sqrt{6} \sqrt{A+\sqrt{A^{2}-2 A+9}+3} / 6\right\} .
$$

Before defining the exponents using (36), we remind the reader about the error exponent region of a MAC introduced in [14, Theorem 4]. The authors show that for a Gaussian MAC with signal powers $S_{1}$ and $S_{2}$ an outer bound on the error exponent region is dictated by three inequalities. The first two error exponents $E_{i}, i=1,2$ are bounded above by $E_{s u}\left(R_{i}, S_{i} / \sigma^{2}\right), i=1,2$ and correspond to the two singleuser error events, and the third exponent $E_{s u}\left(R_{1}+R_{2},\left(S_{1}+\right.\right.$ $\left.S_{2}\right) / \sigma^{2}$ ) corresponds to the joint error event. In all inequalities, $E_{s u}(R)$ represents any upper bound on the reliability function of the single-user discrete-time AWGN channel. Let us denote the three exponents which make use of (36) in the minimization by $F_{1, S h}, F_{2, S h}(\alpha)$ and for the two-user 
component by $F_{12, S h}(\alpha)$. Using the results of [14], the singleuser components are functions of the minimum rate given as (37).

$$
\begin{aligned}
F_{1, S h}^{*} & =2 R_{\text {min }}+E_{s p}\left(\psi\left(R_{\text {min }}\right), A\right) \\
F_{2, S h}^{*}(\alpha) & =F_{1, S h}^{*}-2 \alpha .
\end{aligned}
$$

$F_{12, S h}(\alpha)$ has to be optimized numerically since it does not lend itself to an analytical expression. Using (36) the third exponent as the two-user component is

$$
F_{12, S h}(\alpha)=\min _{R^{\prime} \geq \frac{\alpha}{2}} 2 R^{\prime}-\alpha+E_{s p}\left(\psi\left(2 R^{\prime}\right), A_{1}+A_{2}\right)
$$

where $R^{\prime}=R+\alpha / 2$ and $A_{i}=\mathcal{S}_{i} / \sigma^{2}$ for $i=1,2$. Similarly, the two-user component with the minimum rate is denoted by $F_{12, S h}^{*}(\alpha)$. The derivation of the bounds on the error exponents follow through in the same way as shown in the previous case that makes use of the divergence bound by simply replacing the three exponents in (35) by $F_{1, S h}^{*}, F_{2, S h}^{*}$ and $F_{12, S h}^{*}$.

3) The lower bound by Ashikhmin et al. [13] : As for the third alternative to be used for $E_{u}\left(R_{1}, R_{2}\right)$ we have a more recent result by Ashikhmin et al. [13, Theorem 1] which is a tighter bound on the discrete-time Gaussian reliability function $E(R, A)$ with SNR $A$. We denote it here as $E_{A s h}(R, A)$ and note that it coincides with (36) above a certain code rate. It is, in fact, a convex combination of (36) with a tighter low-rate bound which converges with the zero-rate exponent unlike (36). It does not seem possible to characterize the MSE exponents analytically in the case of the Ashikhmin et al. error exponents. Similarly to the previous case, we denote the three error exponents by $F_{1, A s h}, F_{2, A s h}(\alpha)$ and $F_{12, A s h}(\alpha)$ which are evaluated as

$$
\begin{aligned}
& F_{1, A s h}=\min _{R \geq 0} 2 R+E_{A s h}(R, A) \\
& F_{2, A s h}(\alpha)=F_{1, A s h}-2 \alpha \\
& F_{12, A s h}(\alpha)=\min _{R^{\prime} \geq \frac{\alpha}{2}} 2 R^{\prime}-\alpha+E_{A s h}\left(2 R^{\prime}, A_{1}+A_{2}\right)
\end{aligned}
$$

where $R^{\prime}=R+\alpha / 2$. The optimal values are replaced in (21) to determine the MSE exponents. It should be mentioned that the MSE exponent region in this case may coincide for some choice of SNR with the region based on (36) since the two error exponents coincide for some rates.

In Figure 2, the three outer-bounds on the MSE exponents are numerically evaluated for different values of SNR, which is chosen equal for both transmitters. Clearly, the divergence bound by Nazari is the loosest for all values of SNR whereas the outer bound evaluated using the reliability function bound by Ashikhmin et al. is the tightest. It does seem to coincide with the bound using (36) for high SNR levels in the portion not dominated by the single-user error-event. It is worth mentioning the difference between the performance of the divergence bound and reliability function is most significant for low SNR levels.

\section{CONCLUSIONS}

A new lower bound on any linear combination of the MSE's is derived for two-user parameter-modulation on a discretetime Gaussian MAC without any constraint on the number of signaling dimensions. Additionally, we improved the singleuser results from [4] for the same problem. We then introduced lower bounds on the MSE exponents that could make use of any lower bound on the error exponent of a point-topoint discrete time AWGN channel. The obtained results are numerically evaluated for three different lower bounds on the

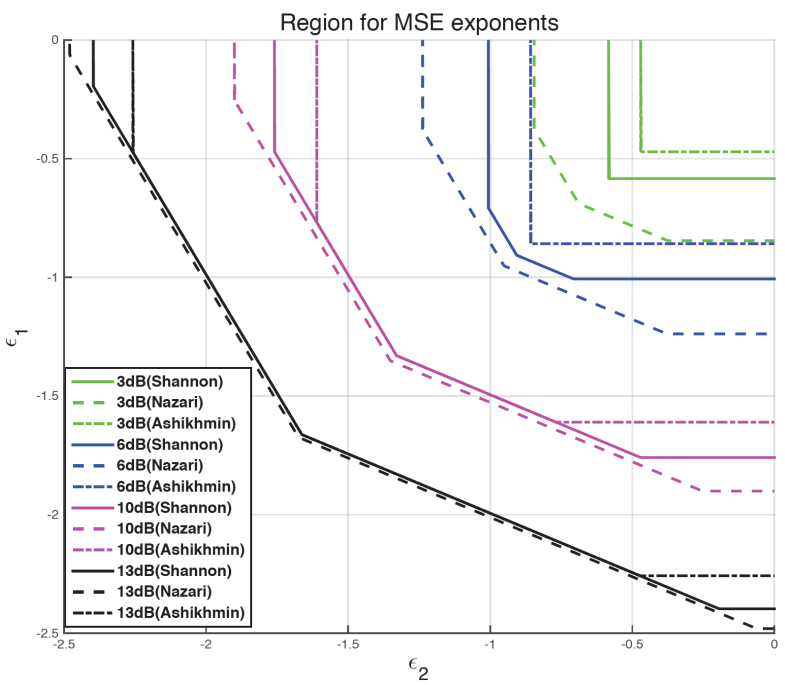

Figure 2. Numerical evaluation of the lower bounds on the error exponents for different values of SNR.

Gaussian reliability function. It is shown that applying the bound from [13] to the MAC can provide a significantly tighter characterization than the sphere-packing bound [11] and the divergence bound [12].

\section{ACKNOWLEDGEMENTS}

The work of EURECOM is supported in part by its industrial members (www.eurecom.fr/en/partners).

\section{REFERENCES}

[1] T. Goblick, "Theoretical limitations on the transmission of data from analog sources," IEEE Transactions on Information Theory, vol. 11, pp. 558-567, October 1965.

[2] A. Wyner and J. Ziv, "On communication of analog data from a bounded source space," The Bell System Technical Journal, vol. 48, pp. 31393172, Dec 1969.

[3] J. Wozencraft and I. M. Jacobs, Principles of Communication Engineering. Wiley, New York, 1965.

[4] N. Merhav, "On optimum parameter modulation-estimation from a large deviations perspective," IEEE Transactions on Information Theory, vol. 58, pp. 7215-7225, December 2012.

[5] J. Ziv and M. Zakai, "Some lower bounds on signal parameter estimation," IEEE Transactions on Information Theory, vol. 15, pp. 386-391, November 1969

[6] D. L. Cohn, "Minimum mean square error without coding," Ph.D. dissertation, MIT, June 1970.

[7] M. V. Burnashev, "On the minimax detection of an inaccurately known signal in a white Gaussian noise background," Theory of Probability and Its Applications, vol. 24, no. 1, pp. 107-119, 1979.

[8] _ "A new lower bound for the $\alpha$-mean error of parameter transmission over white gaussian channel," IEEE Transactions on Information Theory, vol. 30, pp. 23-34, January 1984.

[9] A. Unsal and R. Knopp, "Transmission of correlated Gaussian samples over a Multiple-Access Channel," in CISS2014, IEEE Conference on Information Sciences and Systems, March 19-21, 2014, Princeton, NJ, 032014.

[10] A. Unsal, "Transmission of analog source samples for remote and distributed sensing," Ph.D. dissertation, Telecom ParisTech, Nov. 2014.

[11] C. E. Shannon, "Probability of error for optimal codes in a Gaussian channel," The Bell System Technical Journal, vol. 38, pp. 611-656, May 1959.

[12] A. Nazari, Error Exponent for Discrete Memoryless Multiple-Access Channels. The University of Michigan, Dec. 2011.

[13] A. E. Ashikhmin, A. Barg, and S. N. Litsyn, "A new upper bound on the reliability function of the Gaussian channel," IEEE Transactions on Information Theory, vol. 46, pp. 1945-1961, September 2000.

[14] L. Weng, S. Pradhan, and A. Anastasopoulos, "Error exponent regions for Gaussian broadcast and multiple-access channels," IEEE Transactions on Information Theory, vol. 54, pp. 2919-2942, July 2008. 